\title{
Functional Independent Scaling Relation for ORR/OER Catalysts
}

\author{
Christensen, Rune; Hansen, Heine Anton; Dickens, Colin F.; Nørskov, Jens K.; Vegge, Tejs
}

Published in:

The Journal of Physical Chemistry Part C: Nanomaterials, Interfaces and Hard Matter

Link to article, DOI:

10.1021/acs.jpcc.6b09141

Publication date:

2016

Document Version

Peer reviewed version

Link back to DTU Orbit

Citation (APA):

Christensen, R., Hansen, H. A., Dickens, C. F., Nørskov, J. K., \& Vegge, T. (2016). Functional Independent Scaling Relation for ORR/OER Catalysts. The Journal of Physical Chemistry Part C: Nanomaterials, Interfaces and Hard Matter, 120(43), 24910-24916. https://doi.org/10.1021/acs.jpcc.6b09141

\section{General rights}

Copyright and moral rights for the publications made accessible in the public portal are retained by the authors and/or other copyright owners and it is a condition of accessing publications that users recognise and abide by the legal requirements associated with these rights.

- Users may download and print one copy of any publication from the public portal for the purpose of private study or research.

- You may not further distribute the material or use it for any profit-making activity or commercial gain

- You may freely distribute the URL identifying the publication in the public portal 


\section{Functional Independent Scaling Relation for ORR/OER Catalysts}

Rune Christensen, ${ }^{\dagger}$ Heine A. Hansen, ${ }^{\dagger}$ Colin F. Dickens, $, \sharp, \mathbb{I}$ Jens K. Nørskov,, , Il and Tejs Vegge*,†

$\dagger$ Department of Energy Conversion and Storage, Technical University of Denmark, Fysikvej bld. 309, DK-2800 Kgs. Lyngby, Denmark

$\ddagger$ SUNCAT Center for Interface Science and Catalysis, SLAC National Accelerator Laboratory, 2575 Sand Hill Road, Menlo Park, CA 94025, USA

IDepartment of Chemical Engineering, Stanford University, Stanford, CA 94305, USA

E-mail: teve@dtu.dk

Phone: (+45) 51641787 


\begin{abstract}
A widely used adsorption energy scaling relation between $\mathrm{OH}^{*}$ and $\mathrm{OOH}^{*}$ intermediates in the oxygen reduction reaction (ORR) and oxygen evolution reaction (OER), has previously been determined using density functional theory and shown to dictate a minimum thermodynamic overpotential for both reactions. Here, we show that the oxygen-oxygen bond in the $\mathrm{OOH}^{*}$ intermediate is, however, not well described with the previously used class of exchangecorrelation functionals. By quantifying and correcting the systematic error, an improved description of gaseous peroxide species versus experimental data and a reduction in calculational uncertainty is obtained. For adsorbates, we find that the systematic error largely cancels the $\mathrm{vdW}$ interaction missing in the original determination of the scaling relation. An improved scaling relation, which is fully independent of the applied exchange-correlation functional, is obtained and found to differ by $0.1 \mathrm{eV}$ from the original. This largely confirms, that although obtained with a method suffering from systematic errors, the previously obtained scaling relation is applicable for predictions of catalytic activity.
\end{abstract}




\section{Introduction}

Development of efficient, highly stable, and inexpensive oxygen evolution reaction (OER) and oxygen reduction reaction (ORR) catalysts are key challenges in large scale commercialization of water electrolysers and hydrogen fuel cells, respectively. ${ }^{1-5}$ In these efforts detailed fundamental knowledge of the catalytic processes is required.

In recent years, a number of studies applying Density Functional Theory (DFT) has provided new insight into the fundamentals of OER and ORR. ${ }^{6-13}$ A significant finding is a scaling relation between the adsorption energy of $\mathrm{OH}^{*}$ and $\mathrm{OOH}^{*}$ intermediates found for a large variety of catalysts and catalytic sites. ${ }^{9-12,14-18}$ When expressed in terms of the free energy the scaling relation is $\Delta G_{\mathrm{OOH}^{*}}=\Delta G_{\mathrm{OH}^{*}}+3.2 \pm 0.2 \mathrm{eV}^{9,15}$ with the $3.2 \mathrm{eV}$ offset determined using the RPBE exchangecorrelation functional. ${ }^{19}$ The $3.2 \mathrm{eV}$ free energy difference compares favorably to an experimental free energy difference of $3.4 \mathrm{eV}$ between $\mathrm{OH}^{-}(\mathrm{aq})$ and $\mathrm{OOH}^{-}(\mathrm{aq}) .{ }^{20,21}$ The optimal adsorption free energy difference between $\mathrm{OH}^{*}$ and $\mathrm{OOH}^{*}$ is $2.46 \mathrm{eV}$ for both ORR and OER. ${ }^{9,11,21}$ The scaling relation forces a minimum thermodynamic overpotential, at which all intermediate reaction steps are exergonic, of $(3.2 \pm 0.2 \mathrm{eV}-2.46 \mathrm{eV}) / 2 e=0.4 \pm 0.1 \mathrm{~V}$ for both reactions. Based on the scaling relation, it is possible to use adsorption free energies as predictors for catalyst activity. For ORR, the adsorption free energy of $\mathrm{OH}^{*}$ can be used as predictor with the optimum at (4.92 $\mathrm{eV}-3.2 \pm 0.2 \mathrm{eV}) / 2=0.9 \pm 0.1 \mathrm{eV}$. Catalysts which adsorb $\mathrm{OH}^{*}$ slightly $(\approx 0.1 \mathrm{eV})$ weaker than Pt have been found to display improved activity over Pt. ${ }^{7,22}$ Predictions of catalyst activity based on the $\mathrm{OH}^{*}$ adsorption free energy relative to Pt have been shown to agree well with experimental findings. ${ }^{7,22}$ Similarly, the adsorption free energy difference between $\mathrm{O}^{*}$ and $\mathrm{OH}^{*}$ is used as a descriptor for highest OER activity. ${ }^{11}$ The highest activity is predicted at a difference of $(3.2 \pm 0.2$ $\mathrm{eV}) / 2=1.6 \pm 0.1 \mathrm{eV}$.

It is important to stress that the $\pm 0.2 \mathrm{eV}$ reported for the scaling relation does not refer to the calculation accuracy in any individual calculation, but how well adsorption free energies calculated for a multitude of different surfaces follow the scaling relation. ${ }^{11,12,15,16}$ Although the general trend is a $3.2 \mathrm{eV}$ difference in adsorption energy between $\mathrm{OH}^{*}$ and $\mathrm{OOH}^{*}$, there is notable scatter around 
this "best fit" trend line. The $\pm 0.2 \mathrm{eV}$ is thus an uncertainty introduced by considering the scaling relation as "universal" 11 for all surfaces and not to be confused with the general calculational uncertainty at the applied level of theory. When considering a narrow class of materials such as Pt skins on Pt alloys, the scatter is significantly less than $0.2 \mathrm{eV} .^{12}$

The calculational accuracy for a given calculation is not known a priori. The largest contribution to the calculational uncertainty is expected to be the exchange-correlation functional. This has become increasingly true as electronic structure codes have been developed to the point where results are largely converged across different codes. ${ }^{23}$ The magnitude of the uncertainty related to the exchange-correlation functional can be difficult to estimate although methods for it has been developed as discussed below. ${ }^{24}$ Recently, Deshpande, Kitchin and Viswanathan ${ }^{18}$ have applied these methods to evaluate the calculational uncertainty in studies of heterogeneous ORR catalysts. An extension to the error estimation method is applied here to determine whether systematic errors are present when calculating the adsorption free energy difference between $\mathrm{OH}^{*}$ and $\mathrm{OOH}^{*}$ with the applied class of exchange-correlation functionals. The extended approach has previously been used for identifying systematic errors in calculated energies for $\mathrm{CO}_{2}$ reduction reactions. ${ }^{25}$ This knowledge improved accuracy of calculations and has been applied in studies of $\mathrm{CO}_{2}$ reduction to formic acid. ${ }^{26}$

A major difference between $\mathrm{OH}^{*}$ and $\mathrm{OOH}^{*}$ is the presence of an oxygen-oxygen bond in the $\mathrm{OOH}^{*}$ intermediate. This bond can to some extend be assumed to be similar to the oxygen-oxygen bond in a peroxide. The triplet ground state of molecular oxygen is known to be very difficult to describe using DFT. ${ }^{27}$ Although peroxides are not spin polarized, systematic errors in calculated enthalpies of metal oxides and metal peroxides for several exchange-correlation functionals including the RPBE functional have been attributed to the difference in oxygen ions. ${ }^{28-31}$ We here seek to determine whether a systematic error is indeed present and determine how it influences the $\mathrm{OH}^{*} / \mathrm{OOH}^{*}$ scaling relation and predictions based on it. If a systematic error is present in the description of the oxygen-oxygen bond, the adsorption free energy of $\mathrm{OOH}^{*}$ is likely also to be systematically inaccurate. The previously determined free energy difference between $\mathrm{OH}^{*}$ and 
$\mathrm{OOH}^{*}$, i.e. the scaling relation offset of $3.2 \mathrm{eV}$, will thus also be systematically inaccurate and lead to inaccuracies in predictions of the minimum thermodynamic overpotentials and optimal adsorption free energies. By quantifying the systematic error not only can the calculational uncertainty for a given surface be reduced, but the accuracy of predictions previously made using the scaling relation also be evaluated.

\section{Theoretical Methods}

To test for systematic errors in the description of peroxide species, the enthalpy of reaction is calculated for different reactions with known reaction enthalpies. Eight representative gas phase reactions (Gas Phase Set in Table 1), where a peroxide is formed, have been examined and the enthalpies of reaction compared to experimental values. ${ }^{32}$

Table 1: Reaction Sets

\begin{tabular}{|c|c|c|c|}
\hline \multicolumn{3}{|c|}{ gas phase set } & \multirow{2}{*}{$\begin{array}{c}\text { reac } \\
\text { (1) }\end{array}$} \\
\hline $2 \mathrm{H}_{2} \mathrm{O}$ & $\rightarrow$ & $\mathrm{H}_{2}+\mathrm{H}_{2} \mathrm{O}_{2}$ & \\
\hline $\mathrm{CH}_{3} \mathrm{OH}+\mathrm{H}_{2} \mathrm{O}$ & $\rightarrow$ & $\mathrm{H}_{2}+\mathrm{CH}_{3} \mathrm{OOH}$ & (2) \\
\hline $\mathrm{C}_{2} \mathrm{H}_{5} \mathrm{OH}+\mathrm{H}_{2} \mathrm{O}$ & $\rightarrow$ & $\mathrm{H}_{2}+\mathrm{C}_{2} \mathrm{H}_{5} \mathrm{OOH}$ & (3) \\
\hline $2 \mathrm{CH}_{3} \mathrm{OH}$ & $\rightarrow$ & $\mathrm{H}_{2}+\mathrm{CH}_{3} \mathrm{OOCH}_{3}$ & (4) \\
\hline $\mathrm{C}_{3} \mathrm{H}_{7} \mathrm{OH}+\mathrm{H}_{2} \mathrm{O}$ & $\rightarrow$ & $\mathrm{H}_{2}+\mathrm{C}_{3} \mathrm{H}_{7} \mathrm{OOH}$ & (5) \\
\hline$\left(\mathrm{CH}_{3}\right)_{2} \mathrm{CHOH}+\mathrm{H}_{2} \mathrm{O}$ & $\rightarrow$ & $\mathrm{H}_{2}+\left(\mathrm{CH}_{3}\right)_{2} \mathrm{CHOOH}$ & (6) \\
\hline $2 \mathrm{C}_{2} \mathrm{H}_{5} \mathrm{OH}$ & $\rightarrow$ & $\mathrm{H}_{2}+\mathrm{C}_{2} \mathrm{H}_{5} \mathrm{OOC}_{2} \mathrm{H}_{5}$ & (7) \\
\hline$\left(\mathrm{CH}_{3}\right)_{3} \mathrm{COH}+\mathrm{H}_{2} \mathrm{O}$ & $\rightarrow$ & $\mathrm{H}_{2}+\left(\mathrm{CH}_{3}\right)_{3} \mathrm{COOH}$ & (8) \\
\hline \multicolumn{4}{|c|}{ adsorbate set } \\
\hline $\mathrm{OH}^{*}{ }_{\mathrm{Pt}}{ }^{a}+\mathrm{H}_{2} \mathrm{O}$ & $\rightarrow$ & $\mathrm{H}_{2}+\mathrm{OOH}^{*} \mathrm{Pt}^{a}$ & $(* 1)$ \\
\hline $\mathrm{OH}^{*}{ }_{\mathrm{Ag}}^{b}+\mathrm{H}_{2} \mathrm{O}$ & $\rightarrow$ & $\mathrm{H}_{2}^{2}+\mathrm{OOH}^{*}{ }_{\mathrm{Ag}}^{b}$ & $(* 2)$ \\
\hline $\mathrm{OH}^{*}{ }_{\mathrm{Pd}}^{a}+\mathrm{H}_{2} \mathrm{O}$ & $\rightarrow$ & $\mathrm{H}_{2}+\mathrm{OOH}^{*}{ }_{\mathrm{Pd}}^{a}$ & $(* 3)$ \\
\hline \multicolumn{4}{|c|}{ verification set } \\
\hline $2 \mathrm{CH}_{4}$ & $\rightarrow$ & $\mathrm{H}_{2}+\mathrm{C}_{2} \mathrm{H}_{6}$ & (v1) \\
\hline $\mathrm{CH}_{4}+\mathrm{H}_{2} \mathrm{O}$ & $\rightarrow$ & $\mathrm{H}_{2}+\mathrm{CH}_{3} \mathrm{OH}$ & (v2) \\
\hline $\mathrm{CH}_{4}+\mathrm{C}_{2} \mathrm{H}_{6}$ & $\rightarrow$ & $\mathrm{H}_{2}+\mathrm{C}_{3} \mathrm{H}_{8}$ & (v3) \\
\hline $\mathrm{C}_{2} \mathrm{H}_{6}+\mathrm{H}_{2} \mathrm{O}$ & $\rightarrow$ & $\mathrm{H}_{2}+\mathrm{C}_{2} \mathrm{H}_{5} \mathrm{OH}$ & (v4) \\
\hline
\end{tabular}

In the case of ethyl hydroperoxide, $\mathrm{C}_{2} \mathrm{H}_{5} \mathrm{OOH}$, and n-propyl hydroperoxide, $\mathrm{C}_{3} \mathrm{H}_{7} \mathrm{OOH}$, experimental data obtained with sufficient precision is unavailable. Instead, cross verified enthalpies calculated using quantum chemistry methods, which are well suited for highly accurate description of gaseous molecules, are used for these species. ${ }^{33}$ To test whether errors are related to the forma- 
tion of a peroxide specie, four verification reactions similar to the others but without the formation of peroxide are examined in a similar manner (Verification Set in Table 1). We expect the enthalpy of reaction for the verification reactions to be well-described.

To determine whether systematic errors are not only present in gas phase peroxides, but also for surface bound $\mathrm{OOH}^{*}$, the difference in adsorption enthalpies of $\mathrm{OH}^{*}$ and $\mathrm{OOH}^{*}$ are calculated on different metal surfaces, which span the full range of relevant $\mathrm{OH}^{*} / \mathrm{OOH}^{*}$ adsorption energies for ORR, i.e. the (111) facets of Pt, Ag and Pd. ${ }^{8}$ The enthalpy is calculated for an "Adsorbate Set" (Table 1) of reactions similar to those in the Gas Phase Set. Important aspects such as coverage and electrolyte effects, which should be included to obtain adsorption free energies under realistic ORR/OER conditions, have purposely been disregarded as including these effects will make it difficult to isolate the oxygen-oxygen bond functional dependence.

The reaction enthalpies are calculated with a range of different Generalized Gradient Approximation (GGA) functionals. Here, we focus on the GGA functionals, as this level of functional complexity is often the best applicable for practical trend studies of heterogeneous catalysis. ${ }^{34} \mathrm{Be}-$ sides the RPBE functional ${ }^{19}$ originally used to establish the $\mathrm{OH}^{*} / \mathrm{OOH}^{*}$ scaling relation, we also consider the common GGA functionals $\mathrm{PBE}^{35}$ and BLYP. ${ }^{36,37}$ In addition, three functionals with non-local van der Waals correlation, vdW-DF, ${ }^{38}$ vdW-DF2, ${ }^{39}$ and BEEF-vdW ${ }^{24}$ are included.

The BEEF-vdW functional is constructed with catalysis studies in mind and demonstrated to describe many adsorption energies well. ${ }^{24,40}$ As the BEEF-vdW functional and the associated ensemble are critical to the applied method, it will here be presented in some detail. For a more thorough description of the functional and ensemble the reader is refereed to Wellendorff et al. ${ }^{24}$

The BEEF-vdW exchange-correlation energy is given by

$$
E_{x c}=\sum_{m=0}^{29} a_{m} E_{m}^{\mathrm{GGA}-\mathrm{x}}+\alpha_{c} E^{\mathrm{LDA}-\mathrm{c}}+\left(1-\alpha_{c}\right) E^{\mathrm{PBE}-\mathrm{c}}+E^{\mathrm{nl}-\mathrm{c}}
$$

$E_{m}^{\mathrm{GGA}-\mathrm{x}}$ is the exchange energy obtained with the GGA approximation applying the Legendre polynomial of order $m$ as enhancement factor. $E^{\mathrm{LDA}-\mathrm{c}}$ and $E^{\mathrm{PBE}-\mathrm{c}}$ are LDA and PBE correlation 
energies. The $E^{\mathrm{nl}-\mathrm{c}}$ term is non-local van der Waals correlation identical to that applied in the vdW-DF2 functional.

In a machine learning process, the exchange energy expansion parameters, $a_{m}$, and the correlation weighting, $\alpha_{c}$, are determined such that the functional is optimized to reproduce energies in selected dataset, i.e. formation and reaction energies, reaction barriers, chemisorption energies, van der Waals interactions, and cohesive energies. The optimal parameter values for $a_{m}$ and $\alpha_{c}$ determined in this approach are used for the main BEEF-vdW functional. In addition to the optimal parameters, an ensemble of functionals using sub-optimal parameter values is created in a process inspired by Bayesian statistics. A set of sub-optimal parameters, which reproduces database energies relatively well, is more likely to be accepted into the ensemble of functionals than a worse performing set of parameters.

The ensemble can be used to quantify an estimate of the error and thus also calculational uncertain related to the specific choice of exchange-correlation functional for a given calculation. This is done by computing the energy of interest with a number of ensemble functionals (generally convergence is reached at 2000). ${ }^{24}$ The standard deviation in calculated energies across the ensemble has been shown to be a good quantitative error estimate. ${ }^{24}$ The procedure can be viewed as an improved method for testing the robustness of the results by recalculating with a range of "mindfully selected" functionals, as discussed by Sabbe et al. ${ }^{34}$

All ensemble functionals differ from the main BEEF-vdW functional only in the weight given to the individual energy terms in (1). Once a self-consistent BEEF-vdW calculation has been performed and the energy of each term obtained, the energy for all ensemble functionals can easily be evaluated at single point level accuracy by simply multiplying a matrix containing the parameters $a_{m}$ and $\alpha_{c}$ for the ensemble functionals with a vector containing the individual energy terms. The method does not only help quantify error estimates but is also useful for obtaining energies computed with a large range of functionals very efficiently.

The VASP code using the projector augmented-wave (PAW) method has been used for all calculations. ${ }^{41-44}$ To reduce errors related to choice of PAW potentials, high accuracy hard potentials 
distributed with the code have been used for $\mathrm{H}, \mathrm{O}$, and $\mathrm{C}$. Default potentials have been used for $\mathrm{Pt}$ and $\mathrm{Ag}$, and a potential treating the $4 p$ electrons as valence electrons has been used for Pd (pv potential). A plane wave energy cut-off at $650 \mathrm{eV}$ has been used. Both molecular oxygen and superoxide ions are spin polarized. Although the oxygen-oxygen bonds present in the examined structures are not expected to exhibit spin polarization, calculations allowing spin polarization were performed. See Supporting Information for further calculational details.

\section{Results and discussion}

The enthalpy of reaction has been calculated for the reactions in Table 1 . The errors versus reference data are shown in Table 2 for reactions in the Gas Phase Set and the Verification Set.

Table 2: Errors in calculated $\Delta H_{r}^{\circ}(\mathrm{eV})$ for reactions in Table 1 with different exchangecorrelation functionals.

$\begin{array}{rrrcccc}\text { reac } & \text { PBE } & \text { RPBE } & \text { BEEF-vdW } & \text { vdW-DF } & \text { vdW-DF2 } & \text { BLYP } \\ (1) & 0.25 & 0.29 & 0.26 & 0.41 & 0.44 & 0.35 \\ (2) & 0.19 & 0.24 & 0.22 & 0.36 & 0.40 & 0.30 \\ (3) & 0.21 & 0.24 & 0.22 & 0.36 & 0.39 & 0.29 \\ (4) & 0.17 & 0.18 & 0.21 & 0.36 & 0.39 & 0.25 \\ (5) & 0.21 & 0.24 & 0.24 & 0.39 & 0.41 & 0.31 \\ (6) & 0.21 & 0.23 & 0.21 & 0.37 & 0.42 & 0.27 \\ (7) & 0.14 & 0.14 & 0.19 & 0.34 & 0.36 & 0.21 \\ (8) & 0.24 & 0.25 & 0.27 & 0.42 & 0.46 & 0.33 \\ & & & & & & \\ \text { avg. }^{a} & 0.20 & 0.22 & 0.23 & 0.38 & 0.41 & 0.29 \\ \text { SD }^{b} & 0.03 & 0.04 & 0.02 & 0.03 & 0.03 & 0.04 \\ & & & & & & \\ (\mathrm{v} 1) & 0.01 & -0.01 & -0.01 & 0.00 & -0.05 & -0.04 \\ (\mathrm{v} 2) & 0.05 & 0.05 & 0.06 & 0.11 & 0.10 & 0.07 \\ (\mathrm{v} 3) & -0.03 & -0.06 & -0.03 & -0.03 & -0.08 & -0.10 \\ (\mathrm{v} 4) & 0.02 & 0.00 & 0.03 & 0.08 & 0.08 & 0.03\end{array}$

${ }^{a}$ Average error and ${ }^{b}$ standard deviation for reactions (1)-(8).

The vdW functionals have been adjusted for a previously identified systematic error of 0.09 $\mathrm{eV}$ on the $\mathrm{H}_{2}$ molecule using the BEEF-vdW functional. ${ }^{25,26,45,46}$ This generally decreases the errors. Reactions (1)-(8) have similar errors with a given functional as seen in Table 2, where the average error (avg.) and the standard deviation on the error (SD) are also reported. The verification reactions (v1)-(v4) do not display the same systematic error, indicating a systematic error related to the formation of a peroxide bond. 
The error is consistently above the average in reaction (1) and below the average in reactions (4) and (7). A likely explanation is that the amount of reactant $\mathrm{H}_{2} \mathrm{O}$ is different for reaction (1) (two $\mathrm{H}_{2} \mathrm{O}$ ) and reactions (4) and (7) $\left(\right.$ no $\mathrm{H}_{2} \mathrm{O}$ ) as compared to the other reactions (one $\mathrm{H}_{2} \mathrm{O}$ ). For a given functional, the small variation in error within the Gas Phase Set and the Verification Set can thus largely be explained by an error of $0.02-0.05 \mathrm{eV}$ per $\mathrm{H}_{2} \mathrm{O}$ molecule. This is discussed further below.

Having established that the formation of a peroxide is the dominant reason for the systematic errors, it is examined whether such systematic errors are also present when comparing free energies of $\mathrm{OH}^{*}$ and $\mathrm{OOH}^{*}$ adsorbed on catalytic surfaces, where accurate experimental values remain elusive. Firstly, calculational uncertainties are quantified using the BEEF ensemble. For each reaction, the enthalpy has been calculated with the ensemble of functionals and the standard deviation determined (Table 3). The standard deviation is $0.19 \pm 0.02 \mathrm{eV}$ for reactions in the Gas Table 3: BEEF ensemble standard deviation (eV) before and after correction of the O-O bond.

\begin{tabular}{ccc}
\hline reac & std. dev. & $\begin{array}{c}\text { std. dev. } \\
\text { corrected }\end{array}$ \\
\hline$(1)$ & 0.17 & 0.04 \\
$(2)$ & 0.19 & 0.04 \\
$(3)$ & 0.19 & 0.04 \\
$(4)$ & 0.20 & 0.06 \\
$(5)$ & 0.18 & 0.04 \\
$(6)$ & 0.19 & 0.05 \\
$(7)$ & 0.20 & 0.05 \\
$(8)$ & 0.19 & 0.05 \\
$(* 1)$ & 0.19 & 0.05 \\
$(* 2)$ & 0.19 & 0.04 \\
$(* 3)$ & 0.19 & 0.05 \\
\hline$(\mathrm{v} 1)$ & 0.03 & 0.03 \\
$(\mathrm{v} 2)$ & 0.05 & 0.05 \\
$(\mathrm{v} 3)$ & 0.05 & 0.05 \\
$(\mathrm{v} 4)$ & 0.06 & 0.06 \\
\hline
\end{tabular}

Phase Set and Adsorbate Set and $0.05 \pm 0.02 \mathrm{eV}$ for the reactions in the Verification Set. The functional dependence of the result and thus the calculational uncertainty is found to be signif- 
icantly larger for reactions in the Gas Phase Set and the Adsorbate Set than for reactions in the Verification Set. Further, the functional dependence is seen to be similar in magnitude for the Gas Phase Set reactions and the Adsorbate Set reactions. We further note that $0.19 \mathrm{eV}$ is comparable to the scaling relation offset uncertainty found by Deshpande, Kitchin and Viswanathan ${ }^{18}$ calculated from separate uncertainties on $\mathrm{OH}^{*}$ and $\mathrm{OOH}^{*}$ using statistical measures. It is also worth noting that the ensemble standard deviation is a good quantitative estimate of the errors observed with the BEEF-vdW functional in Table 2, although it is slightly smaller than the on average $0.23 \mathrm{eV}$ error for the Gas Phase Set reactions.

To examine whether the increased functional dependence for the Gas Phase Set and Adsorbate Set is due to the presence of an oxygen-oxygen bond, the effect of applying an energy correction to the oxygen-oxygen bond is examined. For each of the ensemble functionals, the average difference from the electronic energy calculated with the BEEF-vdW functional is determined for the reactions in the Gas Phase Set and the Adsorbate Set and applied as an energy correction for that particular ensemble functional. The energy correction $c_{f}$ for a given ensemble functional $f$ is thus given by

$$
c_{f}=\frac{1}{11} \sum_{r}\left(E_{B E E F-v d W, r}-E_{f, r}\right)
$$

where $r$ is the 11 reactions in the Gas Phase Set and the Adsorbate Set. $E_{B E E F-v d W, r}$ and $E_{f, r}$ are the electronic energies of reaction $r$ calculated with the BEEF-vdW functional and the ensemble functional $f$, respectively. After a correction has been applied to each ensemble functional, $E_{f, r, \text { corrected }}=E_{f, r}+c_{f}$, the standard deviation for each reaction is recalculated using the corrected energies and found to be significantly smaller and in agreement with those observed for the verification reactions (Table 3). It is thus shown to be generally valid for GGA-vdW type functionals that the functional dependence can be minimized effectively through an identical energy correction to the reactions in the Gas Phase Set and the Adsorbate Set and be brought to the level of the 
reactions in the Verification Set. The systematic error causing significant functional dependence observed for the reactions in the Gas Phase Set hence applies identically to the reactions in the Adsorbate Set.

An illustrative way to examine correlations in the functional dependence is by plotting the calculated enthalpies of reactions for two reactions versus each other with several functionals. ${ }^{25}$ In Figure 1, the calculated enthalpies of the gas phase formation of methyl hydroperoxide from methanol (Reaction (2)) is compared to the enthalpy difference between $\mathrm{OH}^{*}$ and $\mathrm{OOH}^{*}$ adsorbed on Pt (Reaction $(* 1))$.

The 2000 functionals in the BEEF ensemble form a straight line with a slope of 1.01 determined through linear regression. The variation in the calculated enthalpy of reaction, is as large as 1.0 $\mathrm{eV}$. The presence of a straight line (coefficient of determination $R^{2}=0.987$ ) with slope 1 indicates that the feature dominating functional dependence is identical in the two reactions. The three primary vdW functionals are placed on or close to this line. The inclusion of vdW interactions will likely stabilize $\mathrm{OOH}^{*}$ more than $\mathrm{OH}^{*}$ due to the size difference. ${ }^{47}$ This explains why the non-vdW functionals deviate slightly from the line towards a larger enthalpy of reaction for Reaction $(* 1)$. To examine this the isolated effect of vdW interactions has been probed by adding vdW interactions to RPBE and PBE using the Tkatchenko-Scheffler ${ }^{48}$ (TS) method using a scaling parameter $s_{R}=0.94$ and reoptimizing geometries. The inclusion of the vdW interactions does not change the gas phase enthalpies significantly, but stabilizes $\mathrm{OH}^{*}$ by $\approx 0.15 \mathrm{eV}$ and $\mathrm{OOH}^{*}$ by $\approx 0.25 \mathrm{eV}$ causing a relative downshift of $\approx 0.10 \mathrm{eV}$ in $\Delta H_{r}^{\circ}$ for reaction $(* 1)$. This downshift brings the non-vdW functionals onto the line.

Similar correlation analyses are made for all the considered reactions. The obtained slopes found through linear regression are depicted in Figure 2. A straight line with a slope close to 1 is obtained for all possible combinations of reactions in the Gas Phase Set and the Adsorbate Set. When reactions from the Verification Set are compared in a similar manner to reactions in the Gas Phase Set and the Adsorbate Set, the results are significantly different. As the functional dependence is much smaller for reactions in the Verification Set, which does not contain peroxide 


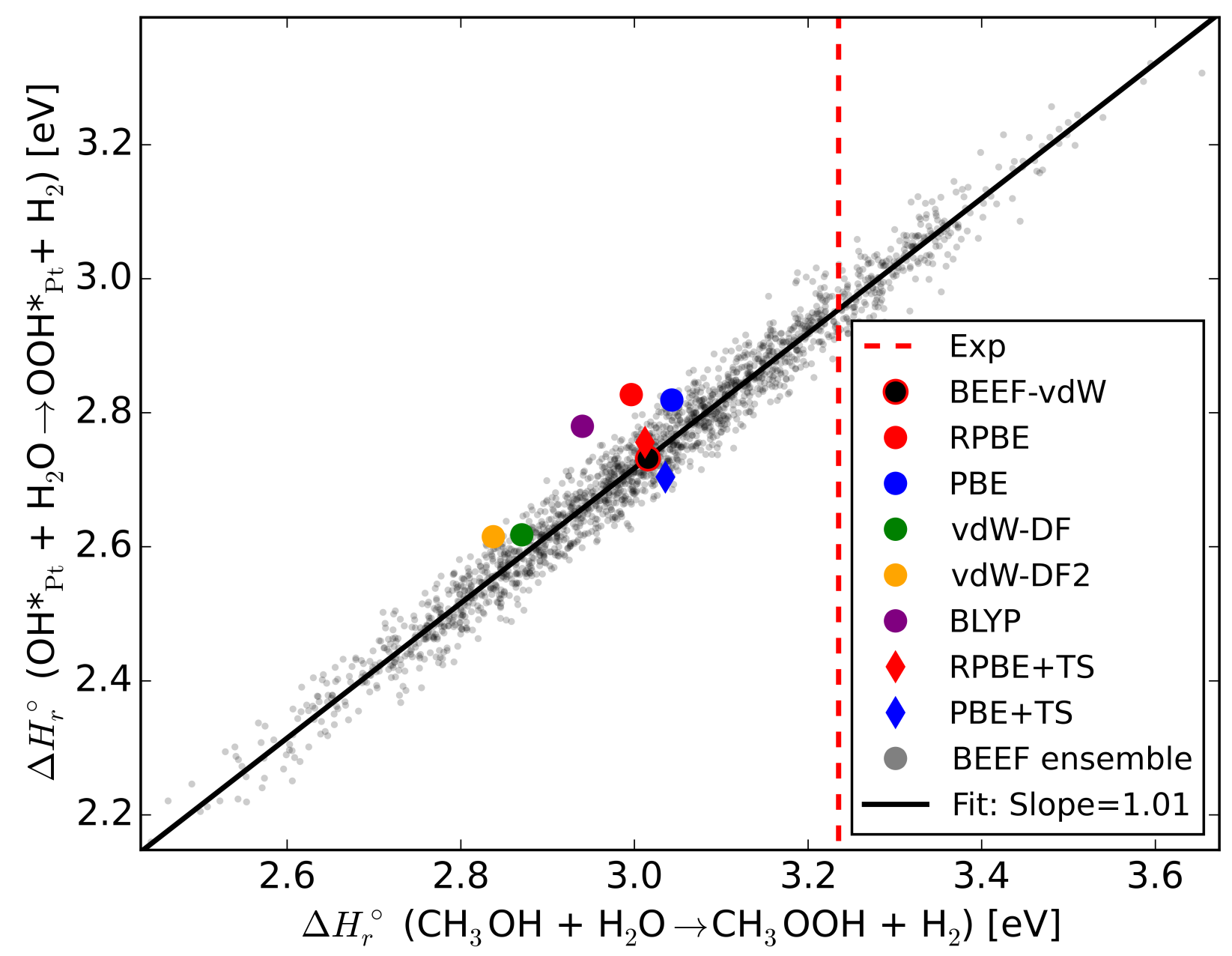

Figure 1: The enthalpies of reaction for Reaction (2) and Reaction $\left({ }^{*} 1\right)$ calculated with various functionals and plotted versus each other. Diamonds mark functionals with added vdW correlation using the Tkatchenko-Scheffler (TS) method. The BEEF-ensemble functionals (semi-transparent grey points) fall on a straight line with a fitted slope of 1.01. The dashed red line marks the experimental enthalpy of Reaction (2).

bonds, the fitted slopes will be large $(>2)$ or close to 0 depending on whether the Verification Set reaction is used as independent or dependent variable, respectively.

In addition to identifying the oxygen-oxygen bond as dominant source of functional dependence and establishing that identical functional dependence is observed for all reactions in the Gas Phase Set and Adsorbate Set, the correlation heat map in Figure 2 can also be used to identify an additional minor contribution to functional dependence. As mentioned above, the trend in errors obtained with the self-consistent functionals (Table 2) suggest that $\mathrm{H}_{2} \mathrm{O}$ can cause a minor 


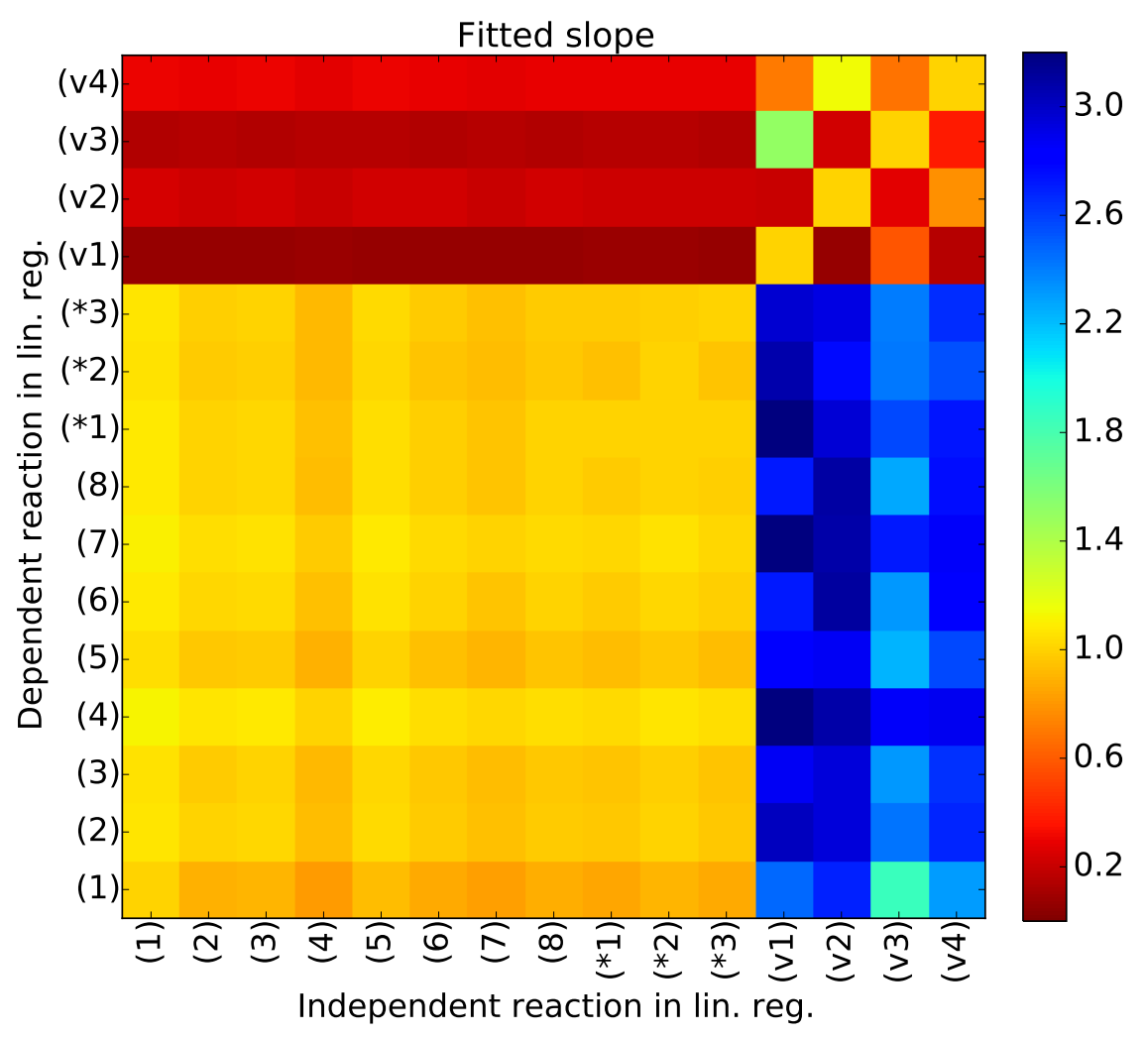

Figure 2: Heat plot depicting the slopes obtain through linear regression on the BEEF ensemble for all possible combinations with the 15 total reactions examined.

error. The same trend with reactions (1), (4), and (7) differing from the rest is observed for the uncorrected standard deviations in Table 3. We examine whether the trend is also reflected in the slopes obtained from linear regression for correlations in the Gas Phase Set as seen in Figure 3. In Figure 3a, the difference between the slope obtained through linear regression and a slope of 1 is depicted. The most significant differences are observed in cases where Reaction (1), (4), or (7) are involved. The pattern fits well with the cause being two reactant $\mathrm{H}_{2} \mathrm{O}$ molecules in Reaction (1), none in Reaction (4) and (7), and one in the other reactions. To test this hypothesis, the slopes are predicted, $a_{\text {predicted }}$, taking the possibility of $\mathrm{H}_{2} \mathrm{O}$ contributing to functional dependence into account.

$$
a_{\text {predicted }}=\frac{1-\alpha n_{\mathrm{H}_{2} \mathrm{O}, x}}{1-\alpha n_{\mathrm{H}_{2} \mathrm{O}, y}} .
$$

$n_{\mathrm{H}_{2} \mathrm{O}, x}$ and $n_{\mathrm{H}_{2} \mathrm{O}, y}$ is the number of $\mathrm{H}_{2} \mathrm{O}$ molecules in the independent and dependent reaction, 


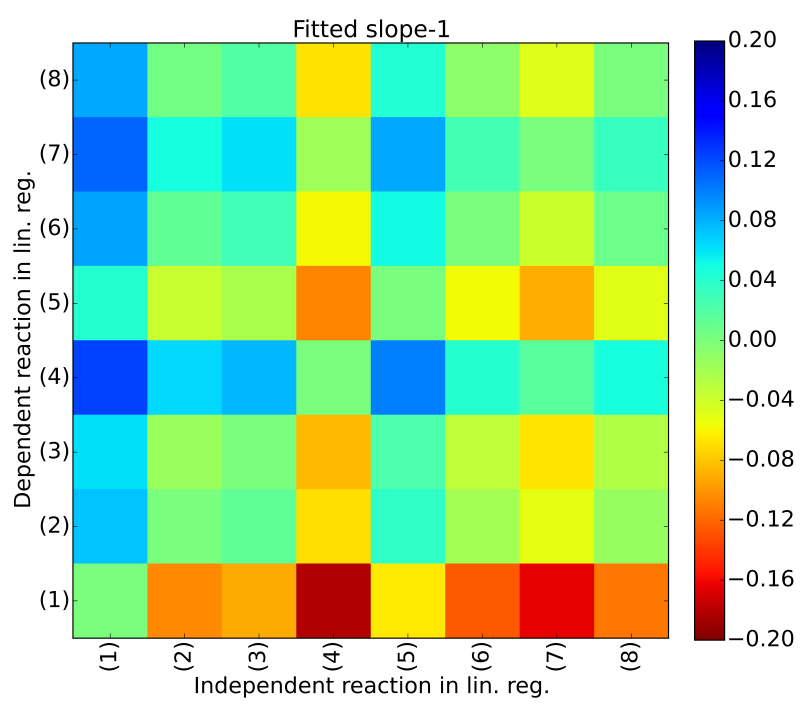

(a)

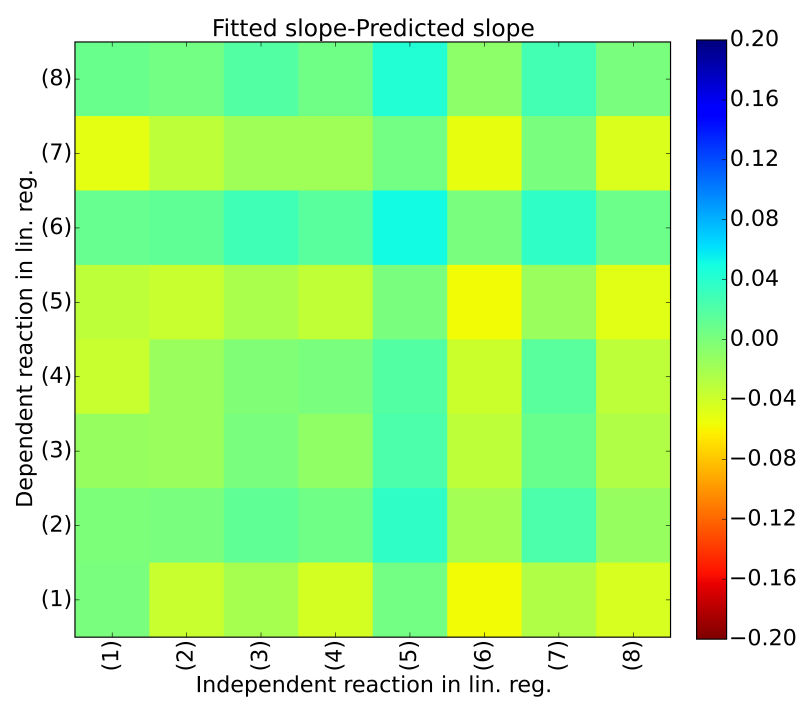

(b)

Figure 3: (a): Difference between the slope found through linear regression and a slope of 1. (b): Difference between the slope found through linear regression and a slope predicted with a $\mathrm{H}_{2} \mathrm{O}$ molecule impacting the slope with $8 \%$ relative to the impact of the peroxide bond.

respectively. $\alpha$ is a parameter which relates the influence of $\mathrm{H}_{2} \mathrm{O}$ on the slope with that of the peroxide bond. In case $\mathrm{H}_{2} \mathrm{O}$ contributes to the functional dependence in a manner which correlates with the functional dependence of the peroxide bond, the optimal value of $\alpha$ is different from 0 . Using $\alpha=-0.08$, the predicted and fitted slopes are very similar for all reactions as observed in Figure $3 b$. Each reactant $\mathrm{H}_{2} \mathrm{O}$ molecule thus influences the slope with an impact of approximately $8 \%$ of that of the peroxide bond. The negative value of $\alpha$ is caused by $\mathrm{H}_{2} \mathrm{O}$ being a reactant molecule and the peroxide bond being present in the product molecule.

The difference between correcting for errors due to the oxygen-oxygen bond and $\mathrm{H}_{2} \mathrm{O}$ independently, as opposed to correcting for a combined total error is insignificant for the purpose of correcting the $\mathrm{OH}^{*} / \mathrm{OOH}^{*}$ scaling relation. This is both due to the common presence of both species in most reactions and the apparent error caused by $\mathrm{H}_{2} \mathrm{O}$ being an order of magnitude smaller than the error caused by the oxygen-oxygen bond.

With correlation in functional dependence between reactions in the Gas Phase Set and Adsorbate Set established, the systematic error of $0.22 \mathrm{eV}$ found for the Gas Phase Set reactions with the RPBE functional can be applied to update the scaling relation between $\mathrm{OH}^{*}$ and $\mathrm{OOH}^{*}$. The lack 
of van der Waals interaction in the RPBE functional is in principle included in the $0.22 \mathrm{eV}$ systematic error for gaseous molecules. However, using the TS method for adding vdW interactions, we found the stabilizing effect to be significant only for adsorbates. This must be considered when transferring the systematic error observed in gas phase to adsorbates. The difference in the missing vdW stabilization for $\mathrm{OH}^{*}$ and $\mathrm{OOH}^{*}, \approx 0.1 \mathrm{eV}$, will to some degree counteract the systematic error on the oxygen-oxygen bond. Based on this, the scaling relation offset can be corrected by 0.1 $\mathrm{eV}$ such that it is now largely functional independent.

The more robust scaling relation is $\Delta G_{\mathrm{OOH}^{*}}=\Delta G_{\mathrm{OH}^{*}}+3.3 \mathrm{eV} \pm 0.2 \mathrm{eV}$. The $\pm 0.2 \mathrm{eV}$ still denotes the uncertainty induced by modeling scattered data with a straight line and not the calculational uncertainty for a given surface. The latter is now quantified and as seen in Table 3 to be reduced from $0.19 \mathrm{eV}$ to $0.05 \mathrm{eV}$ after correction of the systematic error.

Using the functionally independent scaling relation, more robust predictions of minimum overpotentials and optimal adsorption free energies can be performed for a given surface. In general, the minimum thermodynamic overpotential of $\sim 0.4 \mathrm{eV}$ will increase by $0.05 \mathrm{eV}$. Predictions of optimal catalyst reactivities are also modified. For ORR catalysts, the optimal adsorption free energy of $\mathrm{OH}^{*}(\sim 0.8 \mathrm{eV})$ predicted from the improved scaling relation requires a slightly more reactive catalyst, which adsorbs $\mathrm{OH}^{*} 0.05 \mathrm{eV}$ stronger, than previously predicted. For OER, the predicted optimum adsorption free energy difference between $\mathrm{O}^{*}$ and $\mathrm{OH}^{*}(\sim 1.7 \mathrm{eV})$ has increased by 0.05 eV. This requires a slightly less reactive catalyst than previously predicted.

The relatively small changes in optimal adsorption energies and theoretical minimum overpotential will arguably have little impact on the search for new catalysts, especially considering that the scaling relation has only been shown universally valid with $0.2 \mathrm{eV}$ accuracy across different catalytic surfaces. More important is the realization that in spite of being a relatively simple functional approach suffering from a here documented systematic error and lacking description of van der Waals interactions, previous determination of the scaling relation offset based on RPBE calculations has come remarkably close to the functional independent scaling relation offset obtained here. This, in combination with the now quantified and reduced calculational uncertainty, strength- 
ens the applicability of the scaling relation in both prior and future predictions and modeling of catalyst activity.

\section{Conclusions}

We have identified systematic errors on the order of $0.2 \mathrm{eV}$ in the description of peroxide gas phase species using GGA level density functionals. The systematic error was shown to also be present for adsorbed $\mathrm{OOH}^{*}$. It thus impacts the constant free energy difference in the scaling relation previously discovered between adsorption free energies of $\mathrm{OH}^{*}$ and $\mathrm{OOH}^{*}$, which are intermediate adsorbates in both ORR and OER. By identifying and correcting for the systematic error, which for adsorbates was partially canceled by inclusion of van der Waals interaction not previously considered, the calculational uncertainty can be significantly reduced from $0.19 \mathrm{eV}$ to $0.05 \mathrm{eV}$ and a functional independent scaling relation determined. The scaling relation offset is changed from $3.2 \mathrm{eV}$ to $3.3 \mathrm{eV}$. This directly alters predictions of optimal adsorption energies and theoretical minimum potential by $0.05 \mathrm{eV}$. The relative small change from the earlier version of the scaling relation confirms that although derived using a method suffering from systematic errors, the earlier version of the scaling relation is sufficiently accurate to be applicable in catalysis research.

\section{Supporting Information Available}

Supporting Information Available: Computational details, conversion of scaling relation to free energies and specific treatment of error on $\mathrm{H}_{2} \mathrm{O}$

This material is available free of charge via the Internet at http://pubs acs.org/.

\section{Acknowledgement}

The authors thank The Niflheim Linux cluster supercomputer installed at the Department of Physics at the Technical University of Denmark for computational resources This work was supported by 
a research grant (9455) from VILLUM FONDEN (V-SUSTAIN).

\section{References}

(1) Nie, Y.; Li, L.; Wei, Z. Recent Advancements in Pt and Pt-Free Catalysts for Oxygen Reduction Reaction. Chem. Soc. Rev. 2015, 44, 2168-2201.

(2) Trotochaud, L.; Boettcher, S. W. Precise Oxygen Evolution Catalysts: Status and Opportunities. Scr. Mater. 2014, 74, 25-32.

(3) Fabbri, E.; Habereder, A.; Waltar, K.; Kotz, R.; Schmidt, T. J. Developments and Perspectives of Oxide-Based Catalysts for the Oxygen Evolution Reaction. Catal. Sci. Technol. 2014, 4, $3800-3821$.

(4) Cheng, Y.; Jiang, S. P. Advances in Electrocatalysts for Oxygen Evolution Reaction of Water Electrolysis - From Metal Oxides to Carbon Nanotubes. Prog. Nat. Sci.: Mater. Int. 2015, 25, $545-553$.

(5) Debe, M. K. Electrocatalyst Approaches and Challenges for Automotive Fuel Cells. Nature 2012, 486, 43-51.

(6) Keith, J. A.; Jacob, T. In Theory and Experiment in Electrocatalysis; Balbuena, B. P., Subramanian, R. V., Eds.; Modern Aspects of Electrochemistry; Springer New York: New York, NY, 2010; Vol. 50; pp 89-132.

(7) Greeley, J.; Stephens, I. E. L.; Bondarenko, A. S.; Johansson, T. P.; Hansen, H. A.; Jaramillo, T. F.; Rossmeisl, J.; Chorkendorff, I.; Nørskov, J. K. Alloys of Platinum and Early Transition Metals as Oxygen Reduction Electrocatalysts. Nat. Chem. 2009, 1, 552-556.

(8) Nørskov, J. K.; Rossmeisl, J.; Logadottir, A.; Lindqvist, L.; Kitchin, J. R.; Bligaard, T.; Jónsson, H. Origin of the Overpotential for Oxygen Reduction at a Fuel-Cell Cathode. $J$. Phys. Chem. B 2004, 108, 17886-17892. 
(9) Koper, M. T. M. Thermodynamic Theory of Multi-Electron Transfer Reactions: Implications for Electrocatalysis. J. Electroanal. Chem. 2011, 660, 254-260.

(10) Rossmeisl, J.; Logadottir, A.; Nørskov, J. K. Electrolysis of Water on (Oxidized) Metal Surfaces. Chem. Phys. 2005, 319, 178-184.

(11) Man, I. C.; Su, H.-Y.; Calle-Vallejo, F.; Hansen, H. A.; Martínez, J. I.; Inoglu, N. G.; Kitchin, J.; Jaramillo, T. F.; Nørskov, J. K.; Rossmeisl, J. Universality in Oxygen Evolution Electrocatalysis on Oxide Surfaces. ChemCatChem 2011, 3, 1159-1165.

(12) Viswanathan, V.; Hansen, H. A.; Rossmeisl, J.; Nørskov, J. K. Universality in Oxygen Reduction Electrocatalysis on Metal Surfaces. ACS Catal. 2012, 2, 1654-1660.

(13) Halck, N. B.; Petrykin, V.; Krtil, P.; Rossmeisl, J. Beyond the Volcano Limitations in Electrocatalysis - Oxygen Evolution Reaction. Phys. Chem. Chem. Phys. 2014, 16, 13682-13688.

(14) Rossmeisl, J.; Qu, Z.-W.; Zhu, H.; Kroes, G.-J.; Nørskov, J. Electrolysis of Water on Oxide Surfaces. J. Electroanal. Chem. 2007, 607, 83-89.

(15) Zhao, Z.; Zhang, L.; Xia, Z. Electron Transfer and Catalytic Mechanism of Organic Molecule-Adsorbed Graphene Nanoribbons as Efficient Catalysts for Oxygen Reduction and Evolution Reactions. J. Phys. Chem. C 2016, 120, 2166-2175.

(16) Calle-Vallejo, F.; Loffreda, D.; Koper, M. T. M.; Sautet, P. Introducing Structural Sensitivity into Adsorption-Energy Scaling Relations by means of Coordination Numbers. Nat. Chem. 2015, 7, 403-410.

(17) Greeley, J. Theoretical Heterogeneous Catalysis: Scaling Relationships and Computational Catalyst Design. Annu. Rev. Chem. Biomol. Eng. 2016, 7, 605-635.

(18) Deshpande, S.; Kitchin, J. R.; Viswanathan, V. Quantifying Uncertainty in Activity Volcano Relationships for Oxygen Reduction Reaction. ACS Catal. 2016, 6, 5251-5259. 
(19) Hammer, B.; Hansen, L. B.; Nørskov, J. K. Improved Adsorption Energetics within DensityFunctional Theory Using Revised Perdew-Burke-Ernzerhof Functionals. Phys. Rev. B 1999, $59,7413-7421$.

(20) Koper, M. T. M. Theory of Multiple Proton-Electron Transfer Reactions and Its Implications for Electrocatalysis. Chem. Sci. 2013, 4, 2710-2723.

(21) Viswanathan, V.; Hansen, H. A. Unifying Solution and Surface Electrochemistry: Limitations and Opportunities in Surface Electrocatalysis. Top. Catal. 2013, 57, 215-221.

(22) Stephens, I. E. L.; Bondarenko, A. S.; Grønbjerg, U.; Rossmeisl, J.; Chorkendorff, I. Understanding the Electrocatalysis of Oxygen Reduction on Platinum and Its Alloys. Energy Environ. Sci. 2012, 5, 6744-6762.

(23) Lejaeghere, K.; Bihlmayer, G.; Björkman, T.; Blaha, P.; Blügel, S.; Blum, V.; Caliste, D.; Castelli, I. E.; Clark, S. J.; Dal Corso, A. et al. Reproducibility in Density Functional Theory Calculations of Solids. Science 2016, 351.

(24) Wellendorff, J.; Lundgaard, K. T.; Møgelhøj, A.; Petzold, V.; Landis, D. D.; Nørskov, J. K.; Bligaard, T.; Jacobsen, K. W. Density Functionals for Surface Science: Exchange-Correlation Model Development with Bayesian Error Estimation. Phys. Rev. B 2012, 85, 235149.

(25) Christensen, R.; Hansen, H. A.; Vegge, T. Identifying Systematic DFT Errors in Catalytic Reactions. Catal. Sci. Technol. 2015, 5, 4946-4949.

(26) Yoo, J. S.; Christensen, R.; Vegge, T.; Nørskov, J. K.; Studt, F. Theoretical Insight into the Trends that Guide the Electrochemical Reduction of Carbon Dioxide to Formic Acid. ChemSusChem 2016, 9, 358-363.

(27) Jones, R. O.; Gunnarsson, O. The Density Functional Formalism, Its Applications and Prospects. Rev. Mod. Phys. 1989, 61, 689-746. 
(28) Christensen, R.; Hummelshøj, J. S.; Hansen, H. A.; Vegge, T. Reducing Systematic Errors in Oxide Species with Density Functional Theory Calculations. J. Phys. Chem. C 2015, 119, 17596-17601.

(29) Hummelshøj, J. S.; Luntz, A. C.; Nørskov, J. K. Theoretical Evidence for Low Kinetic Overpotentials in Li- $\mathrm{O}_{2}$ Electrochemistry. J. Chem. Phys. 2013, 138, 034703.

(30) Kang, S.; Mo, Y.; Ong, S. P.; Ceder, G. Nanoscale Stabilization of Sodium Oxides: Implications for $\mathrm{Na}-\mathrm{O}_{2}$ Batteries. Nano Lett. 2014, 14, 1016-1020.

(31) Yan, J.; Hummelshøj, J. S.; Nørskov, J. K. Formation Energies of Group I and II Metal Oxides using Random Phase Approximation. Phys. Rev. B 2013, 87, 075207.

(32) NIST Chemistry WebBook, NIST Standard Reference Database Number 69; Linstrom, P. J., Mallard, W. G., Eds.; National Institute of Standards and Technology: Gaithersburg, MD, 2005.

(33) Simmie, J. M.; Black, G.; Curran, H. J.; Hinde, J. P. Enthalpies of Formation and Bond Dissociation Energies of Lower Alkyl Hydroperoxides and Related Hydroperoxy and Alkoxy Radicals. J. Phys. Chem. A 2008, 112, 5010-5016.

(34) Sabbe, M. K.; Reyniers, M.-F.; Reuter, K. First-Principles Kinetic Modeling in Heterogeneous Catalysis: An Industrial Perspective on Best-Practice, Gaps and Needs. Catal. Sci. Technol. 2012, 2, 2010-2024.

(35) Perdew, J. P.; Burke, K.; Ernzerhof, M. Generalized Gradient Approximation Made Simple. Phys. Rev. Lett. 1996, 77, 3865-3868.

(36) Becke, A. D. Density-Functional Exchange-Energy Approximation with Correct Asymptotic Behavior. Phys. Rev. A 1988, 38, 3098-3100.

(37) Lee, C.; Yang, W.; Parr, R. G. Development of the Colle-Salvetti Correlation-Energy Formula into a Functional of the Electron Density. Phys. Rev. B 1988, 37, 785-789. 
(38) Dion, M.; Rydberg, H.; Schröder, E.; Langreth, D. C.; Lundqvist, B. I. Van der Waals Density Functional for General Geometries. Phys. Rev. Lett. 2004, 92, 246401.

(39) Lee, K.; Murray, E. D.; Kong, L.; Lundqvist, B. I.; Langreth, D. C. Higher-Accuracy van der Waals Density Functional. Phys. Rev. B 2010, 82, 081101.

(40) Wellendorff, J.; Silbaugh, T. L.; Pintos, D. G.; Nørskov, J. K.; Bligaard, T.; Studt, F.; Campbell, C. T. A Benchmark Database for Adsorption Bond Energies to Transition Metal Surfaces and Comparison to Selected DFT Functionals. Surf. Sci. 2015, 640, 36-44.

(41) Kresse, G.; Furthmüller, J. Efficient Iterative Schemes for Ab Initio Total-Energy Calculations Using a Plane-Wave Basis Set. Phys. Rev. B 1996, 54, 11169-11186.

(42) Blöchl, P. E. Projector Augmented-Wave Method. Phys. Rev. B 1994, 50, 17953-17979.

(43) Kresse, G.; Joubert, D. From Ultrasoft Pseudopotentials to the Projector Augmented-Wave Method. Phys. Rev. B 1999, 59, 1758-1775.

(44) Klimeš, J.; Bowler, D. R.; Michaelides, A. Van der Waals Density Functionals Applied to Solids. Phys. Rev. B 2011, 83, 195131.

(45) Studt, F.; Abild-Pedersen, F.; Varley, J. B.; Nørskov, J. K. CO and $\mathrm{CO}_{2}$ Hydrogenation to Methanol Calculated Using the BEEF-vdW Functional. Catal. Lett. 2013, 143, 71-73.

(46) Studt, F.; Behrens, M.; Kunkes, E. L.; Thomas, N.; Zander, S.; Tarasov, A.; Schumann, J.; Frei, E.; Varley, J. B.; Abild-Pedersen, F. et al. The Mechanism of $\mathrm{CO}$ and $\mathrm{CO}_{2}$ Hydrogenation to Methanol over Cu-Based Catalysts. ChemCatChem 2015, 7, 1105-1111.

(47) Ramalho, J. P. P.; Gomes, J. R. B.; Illas, F. Accounting for van der Waals Interactions Between Adsorbates and Surfaces in Density Functional Theory Based Calculations: Selected Examples. RSC Adv. 2013, 3, 13085-13100.

(48) Tkatchenko, A.; Scheffler, M. Accurate Molecular Van Der Waals Interactions from GroundState Electron Density and Free-Atom Reference Data. Phys. Rev. Lett. 2009, 102, 073005. 
Graphical TOC Entry

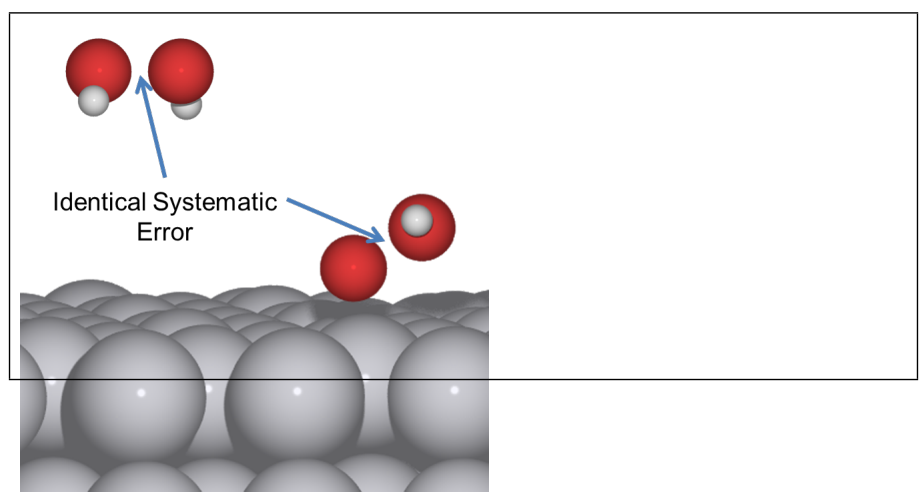

